Cite this: RSC Adv., 2014, 4, 2466

\title{
Self-assemblies of lecithin and $\alpha$-tocopherol as gelators of lipid material
}

\author{
Constantinos V. Nikiforidis ${ }^{* a b}$ and Elke Scholten ${ }^{a b}$
}

Amongst the different mechanisms that have been proposed and used to structure organogels, selfassembly of the gelators into supramolecular structures linked through non-covalent bonds is the most interesting. The gelator activity of LMGOs is often found most effective when micellar or lamellar phases are obtained, which is dependent on the gelator geometry and the specific packing parameter. Gelation can therefore be induced by altering the packing parameter of different gelators, but due to the law restrictions there are only a few edible gelators that can be used to structure edible lipids. Here, we show that a combination of $\alpha$-tocopherol and phosphatidylcholine (PC) can be used to alter the packing geometry to provide supramolecular structures needed for the organogelation. We have observed that when the gelators were combined at 1:1 ratio in sunflower oil, edible organogels were obtained. The firmness of the solid-like material was enhanced when $1.0 \mathrm{wt} \%$ of water was added. The proposed mechanism for this assembly is that most likely cylindrical micellar structures are formed, due to combined assembly of the $\alpha$-tocopherol and phosphatidylcholine, stabilized through physical interactions. Since these interactions, and the accompanied packing geometry, depends on temperature and application of external stresses, the formation of the organogels showed reversibility when the organogels were subjected to shear or when the temperature was increased to values above $35{ }^{\circ} \mathrm{C}$. Polarized microscopy along with small angle X-ray scattering were used to provide a hypothesis for the mechanism behind the gelation.

Received 20th August 2013

Accepted 20th November 2013

DOI: $10.1039 / c 3 r a 46584$ e

www.rsc.org/advances

\section{Introduction}

The texture of complex materials is provided by the structuring of both aqueous and oil phases. ${ }^{1}$ The aqueous phase can mainly be structured by biopolymers, such as proteins and (poly) saccharides, while the physical state of lipid phases mainly depends on the composition of saturated triacylglycerol (TAGs) hardstock. At room temperatures or below, saturated fatty acids crystallise and form a fat crystal network through van der Waals interactions or sintering, providing the lipid phase with solidlike properties. ${ }^{2}$ Saturated (solid) lipids also possess other specific advantageous characteristics over unsaturated (liquid) lipids, such as oxidative stability. These physical and chemical characteristics are important in several lipid-based applications as they determine the texture, spreadability and shelf-life of the condensed material and subsequently also those of the final product. ${ }^{3}$ Additionally, much evidence exist in literature, stating that the intake of saturated and trans fatty acids contribute to global epidemics related to metabolic syndrome and cardiovascular disease (CVD). ${ }^{\mathbf{4} 5}$ As a consequence, legislation limits

${ }^{a}$ Physics and Physical Chemistry of Foods, Wageningen University, P.O. Box 17, 6700AA, Wageningen, The Netherlands

${ }^{b}$ Top Institute Food \& Nutrition, P.O. Box 557, 6700AN Wageningen, The Netherlands. E-mail: costas.nikiforidis@wur.nl; Tel: +31 317485218 the use of trans fats in a variety of products and also consumption of saturated fatty acids is undesired, although their specific health effects are debated. In an attempt to reduce the use and consumption of trans and saturated fatty acids, the use of unsaturated fats (liquid oil) becomes more prominent, and knowledge on how to alter their textural properties of oil is essential. Nowadays, there have been great advances with regards to the nanostructuring of liquid oils into functional fats, also known as organogels. ${ }^{6}$ Structuring oil with alternatives for saturated hardstock is of high interest for many technological and biomedical applications, like food products, ${ }^{3}$ organic electronics ${ }^{7}$ and drug delivery. ${ }^{\mathbf{8}, 9}$

The building blocks for the formation of these organic soft materials (organogels) can vary widely, but mainly fall under the categories of polymeric strands, particle-filled networks and liquid crystalline mesophases. ${ }^{10}$ Some of these building blocks can be formed by self-assemblies of low molecular weight organogelators (LMOGs), and the resulting gels are often described in literature as supramolecular organogels. ${ }^{\mathbf{1 1}}$ The gelator molecules self-assemble into nanofibers (supramolecular polymers) of specific dimensions due to their geometrical packing and then their entanglements create a space-spanning three dimensional network and subsequently entrap solvents in the nanospaces. Electron microscopy images of organogels show a three dimensional network formed by entanglements of 
supramolecular polymers with a variety in size from a few to hundreds of nanometers. ${ }^{12,13}$ The mechanism behind the formation of these structures is the array of monomer units which are linked through non-covalent bonds, such as van der Waals, hydrogen bonding, electrostatic interactions and $\pi-\pi$ or $\tau$-stacking.

These solid-like systems have triggered the interest of scientists due to their functionality caused by the dynamic properties of the supramolecular polymers. The polymers can be reversibly converted to monomers by external stimuli such as temperature, shear, ionic strength, electricity and light. ${ }^{\mathbf{1 4}}$

Despite the high scientific interest, the most effective molecular architecture that can lead to organogels is not exactly known and an exact link between the formed architecture and the molecular properties is not clarified yet. Nevertheless, a variety of organogels have been made, but with potential applications only in pharmaceuticals, organic technology or art conservation..$^{15,16}$ Due to the strict legislations it is very difficult and challenging to find edible gelators for food applications.

In literature there are only a few examples of edible LMOGs. ${ }^{17}$ The most extensively investigated gelators in edible lipid materials are the combination of sterol esters ( $\gamma$-oryzanol) with sterols ( $\beta$-sitosterol), for which the self-assembly into tubular structures is enhanced by mechanical agitation. ${ }^{18,19}$ Other examples of edible LMOGs are long chain saturated fatty acids, saturated fatty alcohols and their mixtures, ${ }^{20,21}$ ricinelaidic $\operatorname{acid}^{22,23}$ and mixtures of lecithin and sorbitan tri-stearate. ${ }^{2}$ All the above components can structure vegetable oils upon formation of certain geometrical packing upon cooling.

In the current work, the potential to structure edible oil with lecithin (phosphatidylcholine, PC) in combination with $\alpha$ tocopherol was investigated (Fig. 1). Lecithin has been thoroughly investigated as a component of non-polar materials for a lot of different potential applications like bioactive compound carriers, $^{24}$ enzyme encapsulation ${ }^{25}$ and in combination with other compounds as edible oil thickeners., ${ }^{2,26}$ According to several researches, purified PC is not able to structure organic solvents, but the presence of impurities like carotenoids ${ }^{27}$ or the addition of small amounts of water or other polar solvents can lead to solid-like materials. ${ }^{28-31}$ When PC is dissolved in nonpolar media, the specific geometry of PC leads to the formation of spherical reverse micelles. The addition of small amounts of a polar solvent induces an uniaxial growth of cylindrical micelles through the formation of hydrogen bonds. The change
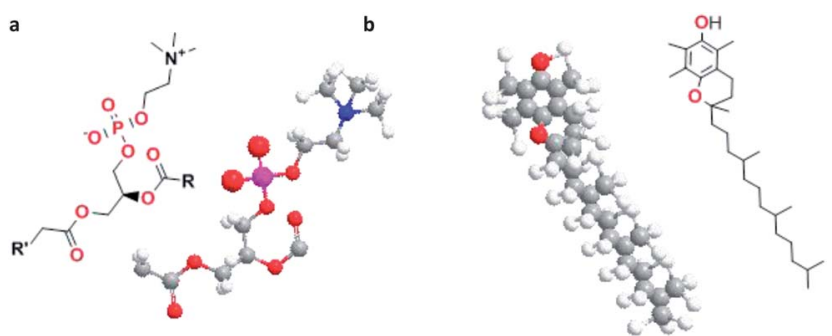

Fig. 1 Molecular structure of PC (a) and $\alpha$-tocopherol (b). With red are indicated the polar areas. in the packing geometry from reverse micelles to reverse cylindrical micelles, leads to the formation of a temporal three dimensional network upon overlap of the cylindrical selfassemblies at a certain threshold concentration. The formed cylindrical self-assemblies of nanofibers (supramolecular polymers) are often referred to in literature as wormlike micelles. ${ }^{32}$

Instead of using a polar solvent to alter the packing geometry of lecithin, we use $\alpha$-tocopherol. $\alpha$-Tocopherol is also known as vitamin $\mathrm{E}$, which has been shown to be inversely associated with mortality from cardiovascular disease at certain concentrations. ${ }^{33}$ The major difference between PC and $\alpha$-tocopherol is their geometry; PC has a large hydrophobic area (tails) and a very small hydrophilic area (head), whereas $\alpha$-tocopherol has a much larger hydrophilic area and a smaller hydrophobic area (Fig. 1). Therefore, their mixtures might lead to a variety of different packing geometries. The formed edible organogels where studied using several techniques, like polarized microscopy, texture analysis and small angle X-ray scattering (SAXS).

\section{Experimental section}

\section{Materials}

Refined sunflower oil was purchased from commercial sources. Its moisture and free fatty acid content was negligible, so it was used without further purification. Oil-free soya lecithin (SOLEC $^{\text {TM }}$, FP30, >30\% phosphatidylcholine) was kindly purchased from Solae (Le Grand-Saconnex, Switzerland). The ethanol soluble material was subjected to purification with a silica column by applying $\mathrm{CH}_{2} \mathrm{Cl}_{2} / \mathrm{MeOH}(1: 1)$ as the eluent. The resulted product consisted of $99 \%$ phosphatidylcholine. All other chemicals where obtained from Sigma-Aldrich (Steinheim, Germany) at the highest degree of available purity.

\section{Sample preparation}

The structuring agent was mixed at ambient temperature with sunflower oil and heated until complete dissolution $\left(80^{\circ} \mathrm{C}, 20\right.$ min). After cooling down they were stored at $5{ }^{\circ} \mathrm{C}$ for 1 week to allow the self-assembly of the structuring agents to take place. The concentrations used were $25 \% \mathrm{w} / \mathrm{w}$ and mixtures of lecithin (PC) and $\alpha$-tocopherol were used in ratios of $1: 1,1: 2$ and $2: 1$. Pure samples of lecithin $(1: 0)$ and $\alpha$-tocopherol $(0: 1)$ were used as references.

\section{Texture analysis}

The TA.XTplus texture analyser (TTC texture technologies, Scarsdale, NY, USA) with a $500 \mathrm{~g}$ load cell was used to analyse the firmness of the gels. A $20 \mathrm{~mm}$ diameter plastic probe was used for compression. Samples were provided in a $50 \mathrm{ml}$ beaker glass, prepared 1 week before use and kept overnight at different temperatures. The sample was compressed until a strain of $50 \%$. The measuring speed was set to $1 \mathrm{~mm} \mathrm{~s}^{-1}$. Tests with 7 replicates indicated that the standard deviation was around 6. For the remaining samples, the average value and the corresponding error margins were calculated from triplicates. 


\section{Microscopy}

The microstructure of the organogels were analysed by cross polarised light microscopy (Reichert-Jung Polyvar, Germany) with Plan $10 \times / 1 \times$ and Plan $40 \times / 1 \times$ objectives at NIZO Food Research, Ede. All gels where kept at $4{ }^{\circ} \mathrm{C}$ for 7 days and their microstructure was observed after storage at ambient temperature for 1 day.

\section{Small angle $X$-rays scattering}

SAXS measurements were performed at the University of Groningen using an advanced Nano-Star SAXS set-up which is a home-made assembly of a NanoStar camera and a Microstar X-ray generator (both by Bruker AXS). The collimation line between the rotating anode generator and the camera consists of multilayer optics Montel-P (by Incoatec) and 3 pinholes (by Rigaku) of $0.5,0.3$ and $0.5 \mathrm{~mm}$ in diameter spaced at distances of $c a .28,58$ and $76 \mathrm{~cm}$ from the middle of the optics unit, respectively. Passing through the optics, the primary beam is monochromized for CuK $\alpha$-radiation (a wavelength of 0.1542 $\mathrm{nm}$ ) and simultaneously collimated to get a low divergent beam (the divergence is below $1 \times 1 \mathrm{mrad}^{2}$ ). Both the optics and the collimation line with the first and the second pinholes are evacuated. The third pinhole located in the sample chamber of the NanoStar camera is in air. All 3 pinholes are manually adjusted. A measured sample, which is also placed in air, is thus located at $c a .80 \mathrm{~cm}$ from the optics, while the sample-todetector distance is $c a .184 \mathrm{~cm}$. The maximal parameters of the rotating anode X-ray generator are $45 \mathrm{kV}$ and $60 \mathrm{~mA}$. At these parameters the flux of the primary X-ray beam at the sample position is estimated to be $c a .8 \times 10^{8}$ photons $\mathrm{s}^{-1} \mathrm{~mm}^{-2}$, while the size of the beam is about $0.4 \mathrm{~mm}$ in diameter. The SAXS intensity profiles were acquired at room temperature $\left(20^{\circ}\right) \mathrm{using}$ an acquisition time of 3 minutes.

\section{Discussion}

\section{Organogel formation}

Our results show that organogels can be created with either crude and purified PC. However, in order to understand the structure formation of the organogels and the accompanying interactions between the different structurants, we aimed to work with pure phosphatidylcholine. As already observed before, different types of impurities may lead to different properties, ${ }^{28}$ and depending on the source of the lecithin, different concentrations of phosphatidylcholine are present. Therefore, in order to obtain reproducible results and to minimize effects of impurities, the phosphatidylcholine fraction from a commercial available food grade soy lecithin was first purified before use.

When purified phosphatidylcholine or $\alpha$-tocopherol was dissolved in sunflower oil, no gel formation was observed at any concentration. However, when phosphatidylcholine or $\alpha$ tocopherol are jointly present in the vegetable oil, at a weight ratio of $1: 1$, gel formation was observed and a spreadable, nonpourable, solid-like material is formed (Fig. 2). It is already well known that pure phosphatidylcholine by itself cannot structure non-polar solvents and its only known combinations that can

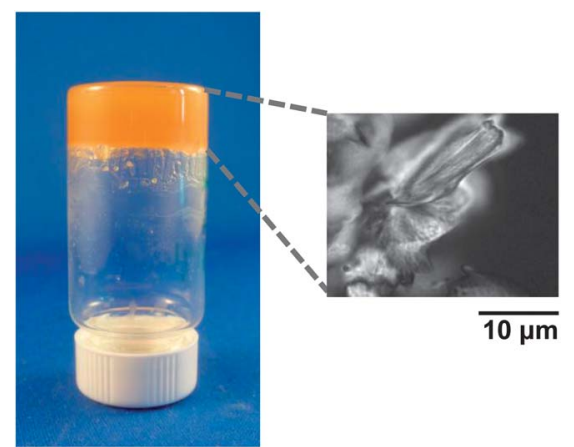

Fig. 2 Image of the non-pourable gel and its microstructure under cross polarized light.

result in lipid material gelation is with sorbitan tri-stearate (STS) or small amounts of a polar solvent.,27,29

In the case of combination of PC with STS, the suggested mechanism is that the building blocks for structure formation are provided by STS crystals. ${ }^{2}$ Lecithin acts as a crystal habit modifier, modifying the packing geometry of the crystal formation and stimulating needle- or plate-shaped crystals. It has to be mentioned at this point that STS molecules have three esterified saturated fatty acids (stearic acid) which are expected to play a key role in the solidification mechanism. In the case of combination of PC with polar solvents, the packing geometry is probably changed by inducing strong hydrogen bonding, which induces an uniaxial growth of the reverse micelles, forming supramolecular polymers. ${ }^{28,29}$

We hypothesize that the crystal formation (Fig. 2) as a result of addition of both PC and $\alpha$-tocopherol, is due to a change in packing geometry of the reverse micelles of lecithin. Formation of assemblies is favourable due to minimization of the hydrophilic head group-solvent interactions, also known as the entropic hydrophobic effect. The shape of the assembly is a result of the geometry of the molecules, and can be estimated taking into account the molecular packing parameter. ${ }^{34,35}$ In the case of lecithin, the small hydrophilic area and the large hydrophobic area favor the formation of small spherical reverse micelles (Fig. 3a). The addition of a larger hydrophilic area in the form of $\alpha$-tocopherol, leads to a gelator mix geometry that would favour packing closer to cylindrical micelles or lamellar phases as depicted in Fig. 3b (decrease in molecular packing parameter).

The spherical reverse micelles do not have the ability to overlap to form space spanning networks. However, the

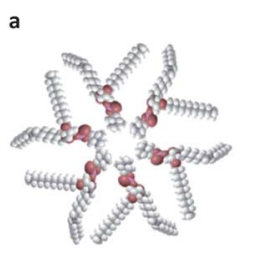

b

Fig. 3 Schematic presentation of the packing geometry. (a) Inverse micelle formation with large curvature (b) formation of small curvature favouring inverse cylindrical micelles or lamellar phases. 
involvement of $\alpha$-tocopherol in the formerly spherical reverse micelles, most likely induce the creation of supramolecular structures, also known as supramolecular polymers that are physically cross-linked and entangled due to non-covalent bonds, such as van der Waals, hydrogen bonding and coordination interactions. This induces the formation of a spanning network and therefore gelation of the system. The high sensitivity of these organogels to shearing (data not shown) indicates that the occurred physical intermolecular interactions are very weak.

\section{Structure stability}

The packing geometry of small molecules is largely dependent on different interactions between the hydrophilic and hydrophobic areas and are therefore often dependent on temperature. Temperature not only changes the interactions, but also changes slightly the solubility of the gelators in the oil. According to the data illustrated in Fig. 4, the formed supramolecular polymers do indeed change with temperature and affects the solid-like behaviour. At low temperatures, there are probably strong physical interactions that promote the specific packing into the supramolecular polymers and the entanglement of these polymers, but at higher temperatures, these interactions weaken and the formation of a network is reduced. At temperatures above $15{ }^{\circ} \mathrm{C}$, the organogel becomes softer and when it reaches $35{ }^{\circ} \mathrm{C}$, the system behaves like a liquid, which probably means that no strong intermolecular interactions between the added molecules are present, but the system is dominated by weak interactions only $(\sim \mathrm{kT})$. Shearing could also promote the breakage of the network (Fig. 5). Actually, this behaviour of gelators is desirable in edible lipid materials. ${ }^{36}$ Since human body temperature and consequently mouth temperature is around $36{ }^{\circ} \mathrm{C}$, lipid materials that show a melting behaviour around this temperature, similar to fats such as milk fat and cocoa butter, have desirable oral perception.

\section{Addition of small amounts of water}

As mentioned previously, PC organogels can also be formed when PC is combined with a small amount of polar solvent like

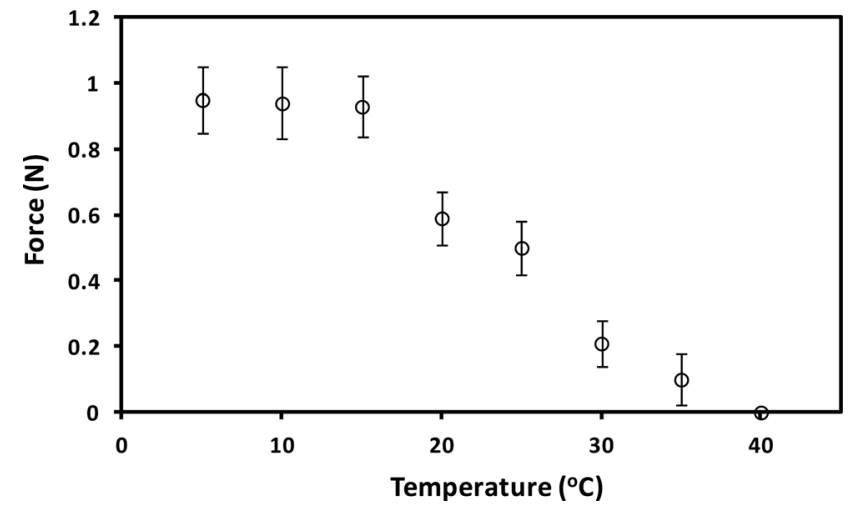

Fig. 4 Effect of storage temperature on the maximal force of $25.0 \mathrm{wt} \%$ total structurant in sunflower oil $(1: 1, \alpha$-tocopherol : PC). The error margins are deduced from duplicates.

\section{Supramolecular polymer}

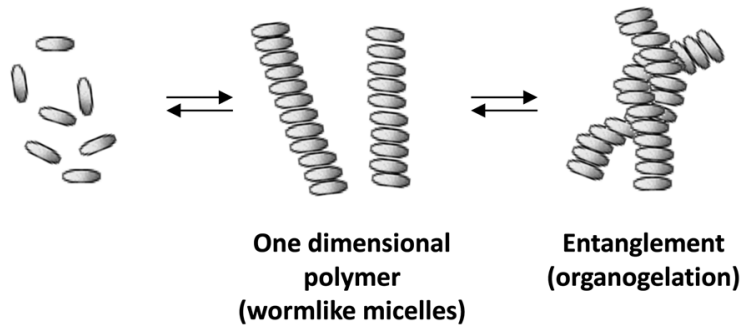

Fig. 5 The reversible formation of supramolecular polymers.

water, glycerol or formamide. The presence of a polar solvent induced the formation of additional hydrogen bonds that enhance the interactions between the PC molecules. ${ }^{28-31}$ The polar molecules, like water, act like a bridge which connects and stabilizes the formed network. The resulted organogels have been observed not to be highly shear sensitive, behaviour that indicates stronger intermolecular interactions when PC is combined with small amounts of a polar solvent. To test whether these interactions could also enhance the gel strength of the formed PC-tocopherol organogels, $1.0 \%(\mathrm{w} / \mathrm{w})$ of water was added to warm sunflower oil solutions containing PC and $\alpha$ tocopherol at different ratios. From all the tested combinations, at the presence of water, only the $1: 1$ and $0: 1$ combinations ( $\alpha$-tocopherol : PC) provided solid-like behaviour after storage for 1 week. On the other hand, in the absence of small amounts of water, only the 1:1 ratio could lead to gel-like structures (Fig. 6). All the other samples were liquids or viscous liquids. The activity of PC with small amounts of water as gelator was already reported before, but it has not been reported for other comparable systems. The present water acts like an intermolecular bridge, connecting the polar regions of PC through hydrogen bonding with the phosphate groups. At the same time a uniaxial growth of "wormlike" reverse micelles is induced. As illustrated in Fig. 7, the presence of $\alpha$-tocopherol (triangles) lead to less strong gels compared to gels prepared with lecithin and water only. In these gels, the $\alpha$-tocopherol molecules are also

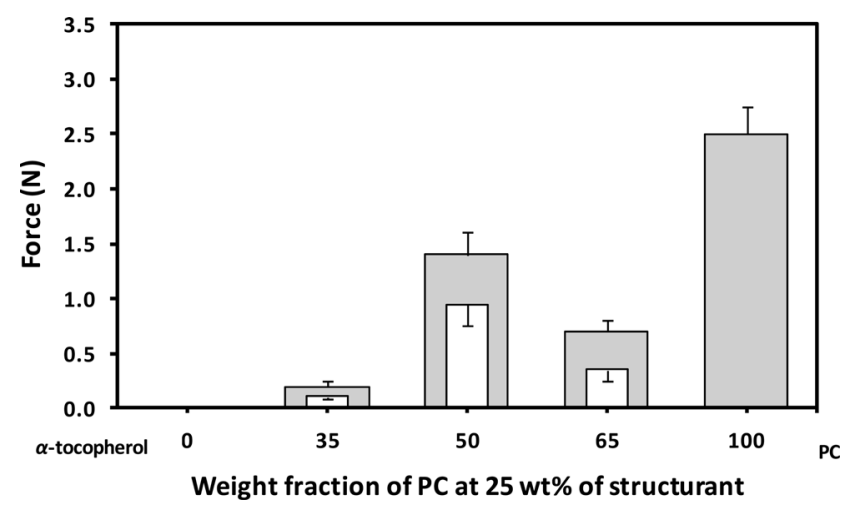

Fig. 6 Maximal force of $25.0 \mathrm{wt} \%$ total structurant with different mass percentage of PC, at the presence ( $\mathbf{\square})$ or absence $(\square)$ of $1.0 \mathrm{wt} \%$ water in sunflower oil. The error margins are deduced from duplicates. 


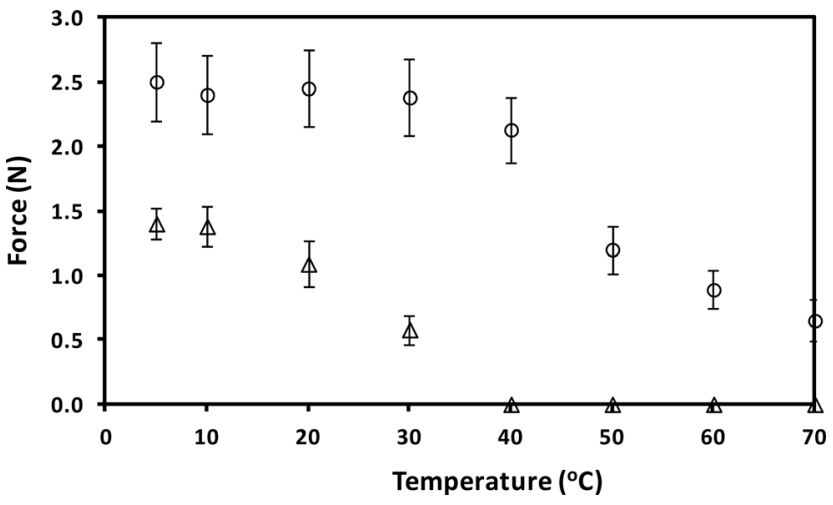

Fig. 7 Comparison of the effect of storage temperature on the maximal force of $25.0 \mathrm{wt} \%$ total structurant in sunflower oil, between PC-water $(\boldsymbol{\square})$ and PC- $\alpha$-tocopherol-water $(\boldsymbol{\Delta})$. The error margins are deduced from duplicates.

involved in the formation of the supramolecular structures, through the interactions of the polar heads. They probably disturb the consecutive row of PC and water molecules connected with hydrogen bonds, thereby reducing the strength of the interactions. Similar behaviour has also been observed when $\beta$-carotene was present. ${ }^{26}$ When $\beta$-carotene was added in $\mathrm{PC} /$ water organogels the viscosity was found to be inversely proportional to the $\beta$-carotene content. According to the authors, $\beta$-carotene acts as an end-cap-active agent in lecithin cylindrical reverse micelles. With the presence of dimethylfloramide (DMFA) the disruption mechanism was more obvious. $^{37-39}$ DMFA molecules are incapable of forming hydrogen bonds with a second PC molecule, which confirms the interruption of the consecutive hydrogen bond chain.

According to the data plotted in Fig. 7, the water molecules induce strong physical interactions between the PC molecules. It seems that even after heating up to $65^{\circ} \mathrm{C}$, the interactions between the molecules are still strong enough to provide a network. At temperatures above $65^{\circ} \mathrm{C}$, the entanglement of the wormlike polymers into long-range orientational order seems to weaken due to a reduced strength of the interactions. At these higher temperature, probably only one dimensional polymers or monomers are present (Fig. 5). Without entanglements of the one-dimensional polymers, these structures do not contribute to the network formation of the building blocks, and behaves more like a viscous liquid.

Adjusting the melting behaviour of lipid material is something desirable. The presence of $\alpha$-tocopherol molecules actually changes the melting profile to lower temperatures. Addition of $\alpha$-tocopherol to weaken the physical interactions between water and lecithin could potentially be used to provide structured lipid materials with different melting behaviour. Fig. 7 illustrates the effect of tocopherol addition to the PC-water systems on the hardness of the gels. The addition of tocopherol leads to a gel hardness roughly 2 times lower than for the gels without tocopherol. When comparing a tocopherol : PC system without water (Fig. 4) and with water (Fig. 7, triangles), we observe an increase (1.5 times) of gel strength upon addition of water. The comparison of Fig. 4 and 7 (triangles) shows that there is only a small shift in the temperature range for which the melting behaviour is observed, which indicates that the melting behaviour is not largely affected by the addition of water. This means that in the presence of water, there are stronger intermolecular interactions, but the entanglement of the supramolecular structures is still affected at similar temperature. The melting behaviour of these organogels, both with and without water, is similar to the behaviour of lipids containing crystalline (saturated) fat, so probably; their mouthfeel could also be similar. Above $40^{\circ} \mathrm{C}$, the samples containing the small amount of water were completely liquid, but when re-cooled and stored, the formed gels had a similar hardness than the initial organogels. This thermal reversibility is often seen for organogels. ${ }^{40}$

\section{Microstructure}

As already discussed previously, the structural assembly of small molecules is partly dependent on the geometry of the molecules and the interactions, first described by the molecular packing parameter by Israelachvili. ${ }^{41}$ Concentration and solvent-molecule interactions are also essential, and nowadays, already a large variety of structural assemblies have been observed. ${ }^{42,43}$ Depending on the PC and water concentration in an organic solvent, different anisotropic or isotropic liquidcrystalline phases have been observed. ${ }^{29}$ The observed anisotropic phases are the reverse nematic $\mathrm{N}_{2}$ phase, the reverse hexagonal $\mathrm{H}_{2}$ phase and the lamellar $\mathrm{L}_{\alpha}$ phases. On the other hand, the possible isotropic phase is a reverse cubic $\mathrm{I}_{2}$ phase. Moreover, at the absence of water and at PC concentrations below $45 \mathrm{wt} \%$, the well-known reverse micellar $\mathrm{L}_{2}$ phase exist. The $\mathrm{L}_{2}, \mathrm{H}_{2}$ and $\mathrm{L}_{\alpha}$ are the dominate phases, whereas $\mathrm{I}_{2}$ and $\mathrm{N}_{2}$ have much more limited stability ranges.

Upon addition of a co-solvent, such as cyclohexane, the interactions between the solvent and the molecules changes and the formation of different structural assemblies can be observed. According to isotherm phase diagrams of the ternary system PC/water/cyclohexane, the system is present as an isotropic reverse micellar $L_{2}$ phase when the concentration of $\mathrm{PC}$ is below $25.0 \mathrm{wt} \%$ and water below $10 \mathrm{wt} \%$. A major part of this phase contains long reverse wormlike micelles that entangle to form a network similar to conventional polymer solutions. When the PC concentration is increased, there is an equilibrium between a reverse hexagonal $\mathrm{H}_{2}$ and a lamellar $\mathrm{L}_{\alpha}$ phase. The formation of these phases results in a high increase of the apparent viscosity. This behaviour is not the same with all organic solvents. When cyclohexane was used, the $\mathrm{H}_{2}$ phase also appeared but only when the total weight fraction $(\varphi)$ of PC and water was between 34.0 and $77.0 \%$. An additional phase, the $\mathrm{N}_{2}$ phase, was observed when the total weight fraction was between 35.0 and $45.0 \%$ and the $\mathrm{L}_{\alpha}$ phase was again observed when the total weight fraction was above $50.0 \%$. It has to be mentioned that at specific PC to water ratios, both $\mathrm{H}_{2}$ and $\mathrm{L}_{\alpha}$ phases are present. $^{32}$

In the current research, where sunflower oil was used as an organic solvent, the concentrations needed to form supramolecular structures seem to be lower. At $1.0 \mathrm{wt} \%$ of water, concentrations of PC above $10 \mathrm{wt} \%$ lead to high viscous liquids 
and above $25 \mathrm{wt} \%$ to solid-like structures, which by default, indicates the presence of $\mathrm{H}_{2}$ and $\mathrm{L}_{\alpha}$ phases. Fig. 8a illustrates images of the PC organogel under polarized light. A mosaic structure can be observed, which could indicate the presence of a lamellar phase $\mathrm{L}_{\alpha}$. However, it is possible that the PC concentration is too low to form lamellar phases, and only reverse micellar $L_{2}$ phases are present. It is most likely that very long wormlike polymers are entangled to form type II hexagonal $\mathrm{H}_{2}$ phases and when the local PC concentration is high enough, also lamellar $\mathrm{L}_{\alpha}$ phases are formed. When PC is combined with $\alpha$-tocopherol at the same total weight fraction, the microstructure is completely different (Fig. 8b). In this image, some
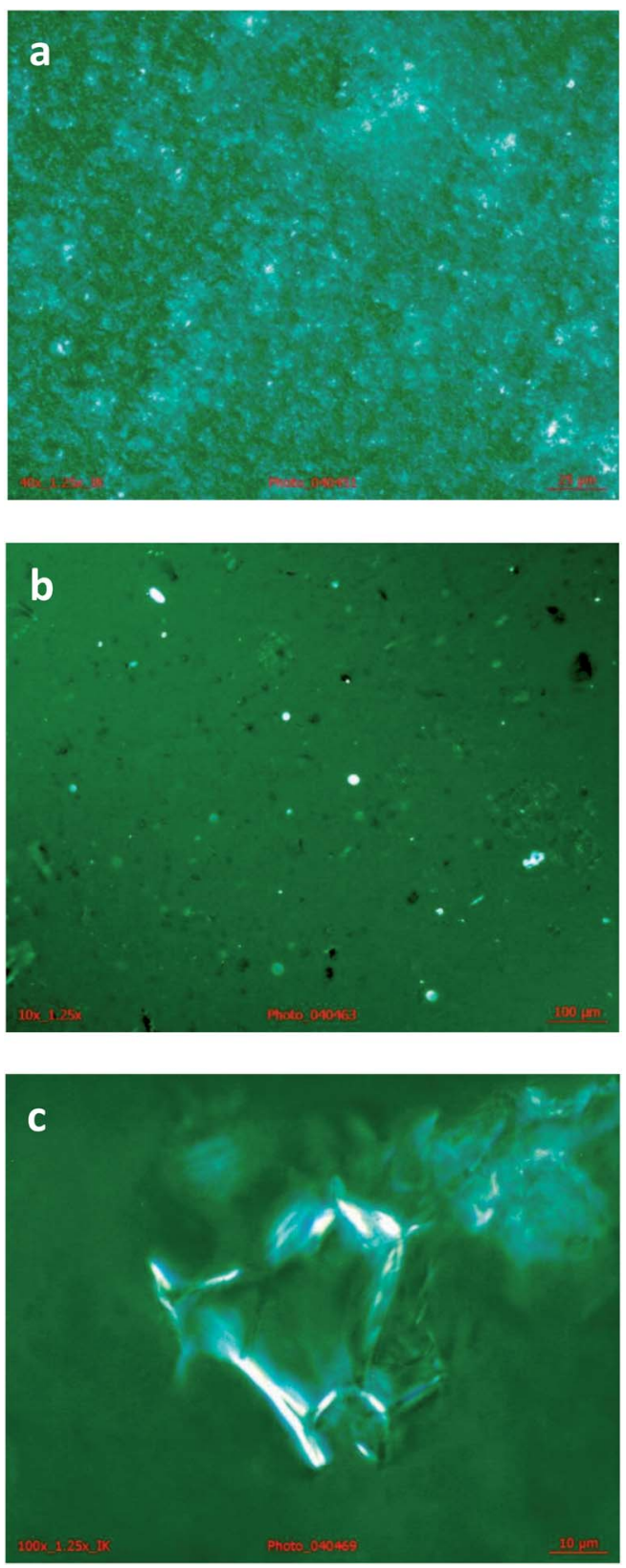

Fig. 8 Micrographs of organogels with $25.0 \mathrm{wt} \%$ of total structurant observed under crossed polarized light at $20^{\circ} \mathrm{C} .24: 1, \mathrm{PC}: \mathrm{H}_{2} \mathrm{O}(\mathrm{a})$, $12: 12: 1, \alpha$-tocopherol : PC : $\mathrm{H}_{2} \mathrm{O}(\mathrm{b}$ and $\mathrm{c})$. isotropic areas can be found, which probably represent the entrapped liquid. Depending on the focus of the microscope, the non-continuous mesophases appear as dark or light coloured areas. It is most likely that the $\alpha$-tocopherol molecules are also involved in the reverse micellar structures and as a consequence the formed supramolecular polymers probably have different dimensions. Focus on one of the formed mesophases showed that the solid-like structure is mainly due to the presence of needle like crystals (Fig. 8c). These structures were similar to the obtained crystals in the absence of small amounts of water (Fig. 2).

In order to gain deeper knowledge on the nature of the supramolecular assemblies observed under the polarized light, $\mathrm{X}$-ray scattering was performed to gain insight in the relevant length scales as illustrated in Fig. 9. It can be clearly seen that when only $\alpha$-tocopherol is present in sunflower oil, there is no structured material in the system. The plotted curve was exactly the same when only PC without added water was present. On the other hand, addition of a very small amount of water (1.0 $\mathrm{wt} \%$ ) resulted in a highly ordered solid-like material, as it revealed from the sharp peak plotted in Fig. 9, at a length scale of around 52 A. Taking into account the length of a single lecithin molecule which can be approximately between 20 and $28 \AA$, this size would be equal to two lecithin molecules. This indeed indicates the presence of either inverse cylindrical micelles with this diameter (size of two lecithin molecules) or a lamellar phase with this width (stacking of two lecithin layers). The ordering of cylindrical micelles may lead to a hexagonal chain packing, similar to the $\alpha$-phase of crystalline triacylglycerides which is the main building block of fat. When PC is combined with $\alpha$-tocopherol and a small amount of water, the peak is broadened and slightly shifted to the left. The broadening and shifting of the peak indicates the formation of slightly larger sizes of the structures and a less ordered system. It can be hypothesized that the main supramolecular structure is still a hexagonal chain packing, but due to the incorporation of the $\alpha$-tocopherol, its size has become slightly larger, and the shape of the reverse cylindrical micelles has slightly changed. However, from these results, we cannot rule out the possibility that lamellar phases are not present.

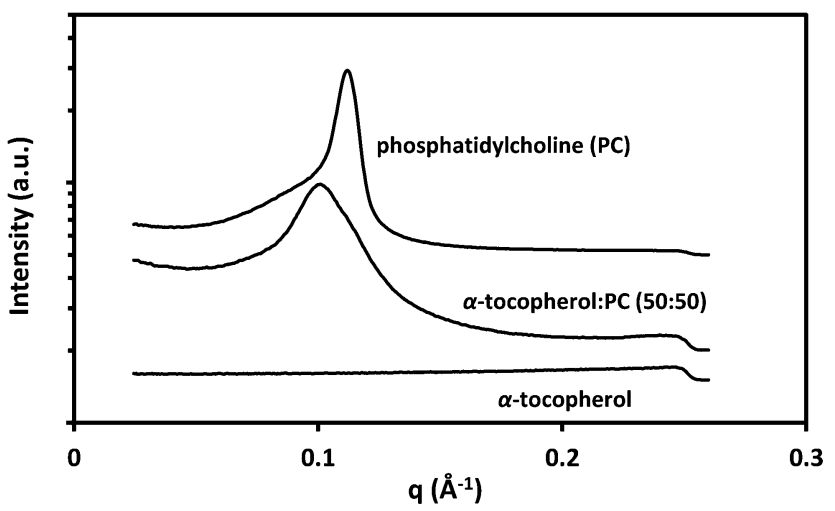

Fig. 9 SAXS patterns of organogels with $25.0 \mathrm{wt} \%$ of total structurant, containing $1.0 w t \%$ of $\mathrm{H}_{2} \mathrm{O}$. 


\section{Conclusions}

In this work we studied the ability of $\alpha$-tocopherol and phosphatidylcholine (PC) to act as gelators of edible lipid material. Addition of these compounds individually in vegetable oil does not yield to any structural organization, but their mixtures at 1: 1 ratio lead to the formation of solid-like structures. A total filler weight fraction above $25 \%$ is needed to induce gelation and the produced organogel is a shear sensitive and thermoreversible gel. At these concentrations and at the absence of $\alpha$-tocopherol, PC molecules form spherical reverse micelles. When $\boldsymbol{\alpha}$-tocopherol molecules are present, they most likely participate in the geometrical packing of the molecules due to interactions between their polar regions, and induce the formation of cylindrical wormlike micelles, which was also confirmed by X-ray analysis. The entanglement of these long supramolecular polymers lead to a more organized systems with soft-solid behaviour.

To change the interactions between the hydrophilic regions of the gelators and thereby influence the molecular assembly, small amounts of water were added to the $\alpha$-tocopherol/PC organogels. When only PC was present, a gum-like organogel was formed with a melting temperature above $65{ }^{\circ} \mathrm{C}$. When PC was combined with $\alpha$-tocopherol, the melting temperature of the thermo-reversible organogels decreased to values around $35{ }^{\circ} \mathrm{C}$, which is desirable when the aim is to form crystalline-free edible lipids. The $\alpha$-tocopherol molecules demote the strong physical interactions in the wormlike micelles, leading to a faster loss of entanglement. Apart from edible applications, the tuneable thermo-reversibility and shear sensitivity of the resulting organogels may also have several applications in pharmaceutical and chemical industry, like development of carriers of biomolecules with high functionality or other uses related to oil technology.

\section{References}

1 D. W. De Bruijne and A. Bot, in Food texture: measurements and perception, ed. A. J. Rosenthal, Aspen, Gaithersburg, 1999.

2 M. Pernetti, K. van Malssen, D. Kalnin and E. Floter, Food Hydrocolloids, 2007, 21, 855-861.

3 M. Pernetti, K. F. van Malssen, E. Floter and A. Bot, Curr. Opin. Colloid Interface Sci., 2007, 12, 221-231.

4 R. P. Mensink, P. L. Zock, A. D. M. Kester and M. B. Katan, Am. J. Clin. Nutr., 2003, 77, 1146-1155.

5 P. L. Zock and R. P. Mensink, Curr. Opin. Lipidol., 1996, 7, 3437.

6 E. D. Co and A. G. Marangoni, J. Am. Oil Chem. Soc., 2012, 89, 749-780.

7 S. S. Babu, S. Prasanthkumar and A. Ajayaghosh, Angew. Chem., Int. Ed., 2012, 51, 1766-1776.

8 S. Sahoo, N. Kumar, C. Bhattacharya, S. S. Sagiri, K. Jain, K. Pal, S. S. Ray and B. Nayak, Des. Monomers Polym., 2011, 14, 95-108.

9 A. Vintiloiu and J. C. Leroux, J. Controlled Release, 2008, 125, 179-192.
10 N. Garti and A. G. Marangoni, in Edible oleogels: structure and health implications, ed. N. Garti and A. G. Marangoni, AOCS Press, Urbana, Illinois, 2011.

11 M. Suzuki and K. Hanabusa, Chem. Soc. Rev., 2010, 39, 455463.

12 N. M. Sangeetha and U. Maitra, Chem. Soc. Rev., 2005, 34, 821-836.

13 P. Dastidar, Chem. Soc. Rev., 2008, 37, 2699-2715.

14 L. Brunsveld, B. J. B. Folmer, E. W. Meijer and R. P. Sijbesma, Chem. Rev., 2001, 101, 4071-4097.

15 H. Tokuyama and Y. Kato, Eur. Polym. J., 2010, 46, 277-282.

16 E. Carretti, L. Dei and R. G. Weiss, Soft Matter, 2005, 1, 17-22.

17 Edible oleogels: structure and health implications, ed. A. G. Marangoni and N. Garti, AOCS Press, Urbana, IL, 2011.

18 A. Bot and W. G. M. Agterof, J. Am. Oil Chem. Soc., 2006, 83, 513-521.

19 A. Bot, R. den Adel, E. C. Roijers and C. Regkos, Food Biophysics, 2009, 4, 266-272.

20 F. G. Gandolfo, A. Bot and E. Floter, J. Am. Oil Chem. Soc., 2004, 81, 1-6.

21 H. M. Schaink, K. F. van Malssen, S. Morgado-Alves, D. Kalnin and E. van der Linden, Food Res. Int., 2007, 40, 1185-1193.

22 A. J. Wright and A. G. Marangoni, J. Am. Oil Chem. Soc., 2006, 83, 497-503.

23 A. J. Wright and A. G. Marangoni, J. Am. Oil Chem. Soc., 2007, 84, 3-9.

24 S. Avramiotis, V. Papadimitriou, E. Hatzara, V. Bekiari, P. Lianos and A. Xenakis, Langmuir, 2007, 23, 4438-4447.

25 M. Mureseanu, A. Galarneau, G. Renard and F. Fajula, Langmuir, 2005, 21, 46484655.

26 V. Clement, G. Savin, J. Jung, R. Mezzenga and M. Leser, Pat., MX2012008439 (A), 2012.

27 P. A. Cirkel, M. Fontana and G. J. M. Koper, Langmuir, 1999, 15, 3026-3028.

28 Y. A. Shchipunov, Usp. Khim., 1997, 66, 328-352.

29 Y. A. Shchipunov, Colloids Surf., A, 2001, 183, 541-554.

30 R. Angelico, A. Ceglie, G. Colafemmina, F. Lopez, S. Murgia, U. Olsson and G. Palazzo, Langmuir, 2005, 21, 140-148.

31 Y. J. Zhao, J. L. Zhang, B. X. Han, C. X. Zhang, W. Li, X. Y. Feng, M. Q. Hou and G. Y. Yang, Langmuir, 2008, 24, 9328-9333.

32 R. Angelico, G. Colafemmina, M. DellaMonica, G. Palazzo, M. Giustini and A. Ceglie, in Trends in Colloid and Interface Science XI, ed. J. B. Rosenholm, B. Lindman and P. Stenius, 1997, 105, 184-191.

33 M. G. Traber, in Modern nutrition in health and disease, ed. M. E. Shils, M. Shike, A. C. Ross, B. Caballero and R. Cousins, Lippincott Williams \& Wilkins, Baltimore, 10th edn, 2006.

34 S. Svenson, J. Dispersion Sci. Technol., 2004, 25, 101-118.

35 K. Van Workum and J. F. Douglas, Phys. Rev. E: Stat., Nonlinear, Soft Matter Phys., 2006, 73, 031502-1-031502-17.

36 B. J. D. Le Reverend, I. T. Norton, P. W. Cox and F. Spyropoulos, Curr. Opin. Colloid Interface Sci., 2010, 15, 84-89.

37 R. Abdel-Rahem and J. Al-Hawarin, J. Dispersion Sci. Technol., 2013, 34, 55-63. 
38 Y. A. Shchipunov and H. Hoffmann, Colloid J., 1998, 60, 794799.

39 Y. A. Shchipunov and H. Hoffmann, Langmuir, 1998, 14, 6350-6360.

40 M. Lescanne, A. Colin, O. Mondain-Monval, F. Fages and J. L. Pozzo, Langmuir, 2003, 19, 2013-2020.
41 J. N. Israelachvili, D. J. Mitchell and B. W. Ninham, J. Chem. Soc., Faraday Trans. 2, 1976, 72, 15251568.

42 J. W. Steed, Chem. Commun., 2011, 47, 1379-1383.

43 S. Wu, J. Gao, T. J. Emge and M. A. Rogers, Soft Matter, 2013, 9, 5942-5950. 